\title{
OBSERVABILITY AND CONTROLLABILITY ANALYSIS FOR SANDWICH SYSTEMS WITH BACKLASH
}

\author{
NA LUO ${ }^{a, b}$, YONGHONG TAN ${ }^{a, *}$, RUILI DONG ${ }^{a}$ \\ ${ }^{a}$ College of Information, Mechanical and Electrical Engineering \\ Shanghai Normal University, 100 Guilin Road, Shanghai, 200234, China \\ e-mail: tany@shnu.edu.cn \\ ${ }^{b}$ School of General Education \\ Sanda University, 2727 Jinhai Road, Shanghai, 201209, China
}

\begin{abstract}
In this paper, an approach to analyze the observability and controllability of sandwich systems with backlash is proposed. In this method, a non-smooth state-space function is used to describe the sandwich systems with backlash which are also non-smooth non-linear systems. Then, a linearization method based on non-smooth optimization is proposed to derive a linearized state-space function to approximate the non-smooth sandwich systems within a bounded region around the equilibrium point that we are interested in. Afterwards, both observability and controllability matrices are constructed and the methods to analyze the observability as well as controllability of sandwich system with backlash are derived. Finally, numerical examples are presented to validate the proposed method.
\end{abstract}

Keywords: backlash, sandwich systems, non-smooth systems, state-space equations, observability, controllability.

\section{Introduction}

Backlash is one of the non-differentiable non-linearities with multi-valued mapping often existing in gear transmissions, hydraulic control valves, ball screw in mechanical transmission mechanisms, etc. (Nordin and Gutman, 2002). The reason for the backlash phenomenon is mainly the gaps existing in gearbox, hydraulic control valves and ball screw, etc.

Usually, backlash does not solely exist in practical systems but connects with other subsystems. For example, in a positioning servo system, a work platform is driven by a gearbox which is driven by a DC motor. This positioning system can be considered a sandwich system with backlash; i.e., the work platform is regarded as a linear subsystem, and the gearbox can be denoted as backlash while the DC motor can also be represented by a linear subsystem. Thus, a sandwich system with backlash is defined as the system where backlash is sandwiched between two linear dynamic subsystems. However, backlash existing in the gearbox of a transmission system may lead to delay, oscillation, undesired steady-state

\footnotetext{
*Corresponding author
}

errors and temporary motion loss due to the transversion of gear teeth trapped in the clearance between mated gear teeth. In this case, the investigation of the observability and controllability of the system at hand should be implemented for the requirement of system analysis and controller design.

It is known that observability and controllability are fundamental properties of a control system, which have been intensively studied in textbooks and papers (Kalman et al., 1969; Sussmann, 1979; van der Schaft, 1982; Isidori, 1989; Klamka, 1991). However, most of these results only focus on smooth linear or non-linear systems (Sontag, 1979; Jank, 2002; Klamka, 2013a; 1975; 2002; 2013b; Isidori, 1989; Herman and Krener, 1977; Balachandran and Shanmugam, 2014; Karthikeyan and Murugesan, 2015). Klamka (2013a) presented a review of controllability problems for a wide class of dynamic systems. Moreover, he also proved sufficient conditions for constrained local controllability for second order dynamical systems with time delay (Klamka, 2013b). Karthikeyan and Murugesan (2015) proposed a method for the analysis of controllability for non-linear stochastic systems with time delays. Furthermore, Balachandran 
and Shanmugam (2014) analyzed the controllability of non-linear implicit fractional integrodifferential systems.

Until today, there have only been several papers considering those properties for non-smooth systems (Koplon and Sontag, 1993; Mincheko and Sirotko, 2002; Murphey and Burdick, 2002; Zhirabok and Shumsky, 2012). Koplon and Sontag (1993) proposed necessary and sufficient conditions for the observability for discrete-time systems with a sign function. On the other hand, Mincheko and Sirotko (2002) proposed a sufficient condition for the local controllability of non-smooth discrete systems with time delay.

Compared with the above-mentioned non-smooth dynamic systems, a sandwich system with backlash contains not only linear dynamic subsystems, but also a backlash non-smooth non-linearity embedded in between. For an observability and controllability analysis of non-linear smooth systems, linearization is one of the options usually applied. However, as backlash is a non-smooth non-linear function, the conventional linearization approach might not be applicable at non-smooth points of backlash non-linearity.

Although there have been some works (Murphey and Burdick, 2002; Zhirabok and Shumsky, 2012) claiming that the controllability and observability analysis methods for linear systems can be extended to systems with non-differentiable components, e.g., the sign function, or friction functions, there is still a lack of a detailed analysis or an investigation of the observability and controllability properties of systems at non-smooth points. Nevertheless, it is very important to know whether the system is observable and controllable at non-smooth points for the design of a proper optimal control strategy for sandwich systems with backlash. Thus, it is indeed worthwhile to solve such problems at non-smooth points of the system. On the other hand, the analysis of the observability and controllability of sandwich systems with backlash will be even more complex since both the input and output of the embedded non-smooth non-linear function are unmeasurable directly. Therefore, it is a real challenge to analyze observability and controllability for those non-smooth sandwich systems.

In this paper, an analysis method for the observability and controllability of sandwich systems with backlash is proposed. Within a small neighborhood of an equilibrium point, a generalized linearization is implemented. Thus, a linearized state space model is constructed to approximate the non-smooth system with backlash around the equilibrium point. Through the investigation of the derived observability and controllability matrices, necessary and sufficient conditions for state observability and controllability are obtained. However, the observability and controllability of the obtained linearized systems are only identical with the observable and controllable subspaces of the sandwich system with backlash within a deterministically local region.

The paper is organized as follows. In Section 2, an input-output sandwich model of backlash is introduced. Then, based on this model, the corresponding non-smooth state-space equation to describe the sandwich system with backlash is developed. Subsequently, a linearized state-space model is derived by a method of generalized gradient-based linearization. In Section 3, both observability and controllability matrices of the linearized system are constructed. Afterwards, observability and controllability for the system based on the observability and controllability matrices is analyzed in Section 4. Then, numerical examples are presented in Section 5 to validate the proposed method. Finally, conclusions are given.

\section{Description of the sandwich model with backlash}

The corresponding architecture of the so-called sandwich system with backlash is shown in Fig. 1. In this system, both $L_{1}$ and $L_{2}$ are the input and output linear dynamic subsystems, respectively, and $N(\cdot)$ represents the backlash subsystem embedded in between $L_{1}$ and $L_{2}$. It is assumed that both $u(k)$ and $y(k)$, i.e., the input and output of the system, are measurable while the internal variables, i.e., $h(k)$ and $v(k)$, are unmeasureable directly. Therefore, the mapping between $u(k)$ and $y(k)$ is a composition mapping due to the involved internal variables, i.e., $h(k)$ and $v(k)$.

Note that the backlash embedded in the sandwich system shown in Fig. 1 is specified by the slopes $m_{1}$ and $m_{2}$ as well as the absolute thresholds $D_{1}$ and $D_{2}$, where $0 \leq m_{1} \leq+\infty, 0 \leq m_{2} \leq+\infty, 0 \leq D_{1} \leq+\infty$, and $0 \leq D_{2} \leq+\infty$. Hence, the discrete-time submodel of backlash is described as

$$
v(k)= \begin{cases}m_{1}\left(h(k)-D_{1}\right) & \text { if } h(k) \geq \frac{v(k-1)}{m_{1}}+D_{1} \\ & \text { and } h(k)>h(k-1) \\ & \text { (increase zone), } \\ v(k-1) & \text { if } \frac{v(k-1)}{m_{2}}-D_{2}<h(k) \\ & <\frac{v(k-1)}{m_{1}}+D_{1} \\ & (\text { memory zone), } \\ & \text { if } h(k) \leq \frac{v(k-1)}{m_{2}}-D_{2} \\ m_{2}\left(h(k)+D_{2}\right) & \text { and } h(k)<h(k-1) \\ & \text { (decrease zone). }\end{cases}
$$




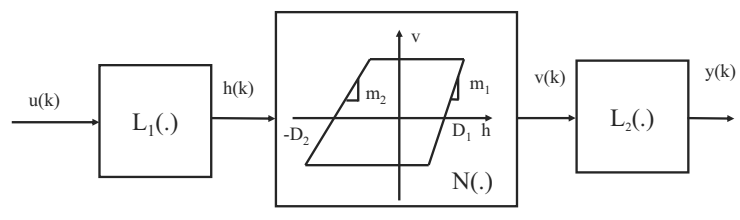

Fig. 1. Structure of a sandwich system with backlash.

submodel of backlash can be rewritten as (Dong et al., 2012)

$$
\begin{aligned}
m(k)= & m_{1}+\left(m_{2}-m_{1}\right) g(k), \\
\tau(k)= & m(k)(h(k)+g(k) h(k) \\
& \left.-D_{1} g_{1}(k)+D_{2} g_{2}(k)\right), \\
v(k)= & \tau(k)+[v(k-1)-\tau(k)] \\
& \times\left(g_{1}(k)-1\right)\left(g_{2}(k)-1\right),
\end{aligned}
$$

where the switching functions $g(k), g_{1}(k)$, and $g_{2}(k)$ are, respectively, defined as

$$
\begin{gathered}
g(k)= \begin{cases}0 & \text { if } \Delta h(k)>0, \\
1 & \text { if } \Delta h(k) \leq 0,\end{cases} \\
g_{1}(k)= \begin{cases}1 & \text { if } h(k)>\frac{v(k-1)}{m_{1}}+D_{1} \text { and } \\
& h(k)>h(k-1), \\
0 & \text { otherwise, }\end{cases} \\
g_{2}(k)= \begin{cases}1 & \text { if } h(k)<\frac{v(k-1)}{m_{2}}-D_{2} \text { and } \\
& h(k)<h(k-1), \\
0 & \text { otherwise, }\end{cases}
\end{gathered}
$$

where $\Delta h(k)=h(k)-h(k-1)$.

Remark 1. The functions of (3)-(5) introduced into the submodel of backlash are just used to represent the change conditions in the operation zones of the backlash subsystem.

Moreover, in this sandwich system, the linear dynamic subsystems, i.e., $L_{1}$ and $L_{2}$, are, respectively, described by

$$
L_{1}:\left\{\begin{array}{l}
x_{1}(k+1)=A_{1} x_{1}(k)+B_{1} u(k), \\
h(k)=C_{1} x_{1}(k)
\end{array}\right.
$$

and

$$
L_{2}:\left\{\begin{array}{l}
x_{2}(k+1)=A_{2} x_{2}(k)+B_{2} v(k), \\
y(k)=C_{2} x_{2}(k)
\end{array}\right.
$$

where $A_{i} \in \mathbb{R}^{n_{i} \times n_{i}}, B_{i} \in \mathbb{R}^{n_{i} \times 1}, C_{i} \in \mathbb{R}^{1 \times n_{i}}, x_{i} \in$ $\mathbb{R}^{n_{i} \times 1}, u \in \mathbb{R}^{1 \times 1}, v \in \mathbb{R}^{1 \times 1}, h$ and $y \in \mathbb{R}^{1 \times 1}$.

Thus, (1)-(7) represent the model describing the sandwich system with backlash. With them, we can obtain the following state-space equations for the sandwich system with backlash as:

$$
\sum:\left\{\begin{array}{l}
x_{1}(k+1)=A_{1} x_{1}(k)+B_{1} u(k), \\
x_{2}(k+1)=A_{2} x_{2}(k)+B_{2} v(h(k))
\end{array}\right.
$$

and

$$
y(k)=\left(\begin{array}{ll}
0 & C_{2}
\end{array}\right)\left(\begin{array}{l}
x_{1}(k) \\
x_{2}(k)
\end{array}\right) .
$$

Suppose that there exists a steady state value of input $u^{*}$, which can maintain the equilibrium point at $\left(\begin{array}{ll}x_{1}^{*} & x_{2}^{*}\end{array}\right)^{T}$ with $h^{*}$ as the corresponding output value of $L_{1}$. Then, there exist the corresponding state-space equations at the equilibrium point are

$$
\left\{\begin{array}{l}
x_{1}^{*}(k+1)=A_{1} x_{1}^{*}(k)+B_{1} u^{*}(k) \\
x_{2}^{*}(k+1)=A_{2} x_{2}^{*}(k)+B_{2} v\left(h^{*}(k)\right) .
\end{array}\right.
$$

Defining the incremental variables as

$$
\begin{aligned}
\hat{u} & =u-u^{*}, \\
\hat{x}_{1} & =x_{1}-x_{1}^{*}, \\
\hat{x}_{2} & =x_{2}-x_{2}^{*}, \\
\hat{h} & =h-h^{*}
\end{aligned}
$$

results in

$$
\left\{\begin{array}{l}
\hat{x}_{1}(k+1)=A_{1} \hat{x}_{1}(k)+B_{1} \hat{u}(k), \\
\hat{x}_{2}(k+1)=A_{2} \hat{x}_{2}(k)+B_{2}\left[v(h(k))-v\left(h^{*}(k)\right)\right]
\end{array}\right.
$$

Consider the backlash function $v(\cdot)$ to be locally Lipschitz continuous on an open set $R$. Then, based on $D_{v}$, which denotes the set of all $h$ where $v$ admits a Fréchet derivative $v^{\prime}(h) \in R$, according to Rademacher's theorem (Rockafellar and Wets, 1998), $R \backslash D_{v}$ has zero Lebesgue measure. Hence, the corresponding Clarke generalized Jacobian $\partial v(h)$ of $v$ at $h^{*}$ is defined as

$$
\partial v\left(h^{*}\right)=\operatorname{conv}\left\{\lim \nabla v(h): h \rightarrow h^{*}\right\}
$$

where $\partial v\left(h^{*}\right)$ is a non-empty, convex and compact set for any fixed $h^{*}$ (Clarke, 1983). Note that Qi and Sun (1993) proved that

$$
v(h(k))=v\left(h^{*}(k)\right)+\partial^{*} v \cdot \hat{h}+o(\|\hat{h}\|)
$$

as $\|\hat{h}\| \rightarrow 0$, where $\partial^{*} v \triangleq a \in \partial v\left(h^{*}\right)$. 
By (1), we obtain

$$
\partial v\left(h^{*}\right)= \begin{cases}\left\{m_{1}\right\} & \text { in increase zones } \\ \left\{0, m_{1}\right\} & \text { but } h^{*}>\frac{v(k-1)}{m_{1}}+D_{1}, \\ \left\{m_{2}\right\} & \text { in increase zones } \\ & \text { in decrease zones } \\ \left\{0, m_{2}\right\} & \text { but } h^{*}<\frac{v(k-1)}{m_{2}}-D_{2}, \\ & \text { in decrease zones } \\ \{0\} & \text { but } h^{*}=\frac{v(k-1)}{m_{2}}-D_{2}, \\ & \text { in memory zones }\end{cases}
$$

This suggests that in a small neighborhood of $h^{*}(k)$ we can approximate $v(h(k))-v\left(h^{*}(k)\right)$ by the generalized gradient based linearization $\partial^{*} v \cdot \hat{h}$. Actually, because of the piecewise linear characteristic of backlash, this approximation is accurate. That is,

$$
\partial^{*} v \hat{h}=v(h(k))-v\left(h^{*}(k)\right) .
$$

So, in terms of (11), we have

$$
\left\{\begin{array}{l}
\hat{x}_{1}(k+1)=A_{1} \hat{x}_{1}(k)+B_{1} \hat{u}(k), \\
\hat{x}_{2}(k+1)=A_{2} \hat{x}_{2}(k)+B_{2} \partial^{*} v \hat{h} .
\end{array}\right.
$$

Since $\hat{h}=C_{1}\left(x_{1}-x_{1}^{*}\right)=C_{1} \hat{x}_{1}$, the state-space equations for the sandwich system with backlash by linearization are

$$
\sum:\left\{\begin{array}{l}
\hat{x}_{1}(k+1)=A_{1} \hat{x}_{1}(k)+B_{1} \hat{u}(k) \\
\hat{x}_{2}(k+1)=A_{2} \hat{x}_{2}(k)+B_{2} \partial^{*} v \cdot C_{1} \hat{x}_{1}
\end{array}\right.
$$

and

$$
\hat{y}(k)=\left(\begin{array}{ll}
0 & C_{2}
\end{array}\right)\left(\begin{array}{c}
\hat{x}_{1}(k) \\
\hat{x}_{2}(k)
\end{array}\right) .
$$

That is,

$$
\sum:\left\{\begin{array}{l}
\hat{x}(k+1)=A \hat{x}(k)+B \hat{u}(k), \\
\hat{y}(k)=C \hat{x}(k),
\end{array}\right.
$$

where

$$
\begin{gathered}
\hat{x}(k+1)=\left(\begin{array}{c}
\hat{x}_{1}(k+1) \\
\hat{x}_{2}(k+1)
\end{array}\right) \in \mathbb{R}^{n \times 1}, \\
A=\left(\begin{array}{cc}
A_{1} & O \\
B_{2} \partial^{*} v C_{1} & A_{2}
\end{array}\right) \in \mathbb{R}^{n \times n}, \\
B=\left(\begin{array}{c}
B_{1} \\
O
\end{array}\right) \in \mathbb{R}^{n \times 1}, \\
C=\left(\begin{array}{ll}
0 & C_{2}
\end{array}\right) \in \mathbb{R}^{1 \times n}, \quad n=n_{1}+n_{2} .
\end{gathered}
$$

\section{Observability and controllability matrices}

It is well known that the analysis of observability and controllability for a linearized system usually depends upon the rank analysis of the corresponding observability and controllability system matrices. For the linearized state-space function of the sandwich system with backlash, which is described by (19), the corresponding observability and controllability matrices can be derived as follows.

3.1. Controllability matrix. Define $M$ as the controllability matrix:

$$
\begin{aligned}
& M=\left(\begin{array}{lllll}
B & A B & A^{2} B & \ldots & A^{n-1} B
\end{array}\right) \\
& =\left(\begin{array}{ccccc}
B_{1} & A_{1} B_{1} & A_{1}^{2} B_{1} & \ldots & A_{1}^{n-1} B_{1} \\
O & H_{1} & H_{2} & \ldots & H_{n-1}
\end{array}\right) \\
& =\left(\begin{array}{ccc}
B_{1} & A_{1} B_{1} & A_{1}^{2} B_{1} \\
O & a B_{2} C_{1} B_{1} & a B_{2} C_{1} A_{1} B_{1}+A_{2} H_{1}
\end{array}\right. \\
& \left.\begin{array}{cc}
\ldots & A_{1}^{n-1} B_{1} \\
\ldots & a B_{2} C_{1} A_{1}^{n-2} B_{1}+A_{2} H_{n-2}
\end{array}\right),
\end{aligned}
$$

where $H_{i}(i=1,2, \ldots, n-1)$ is the corresponding subblock of $M$. Was have

$$
\left\{\begin{array}{l}
H_{i}=a B_{2} C_{1} A_{1}^{n-2} B_{1}+A_{2} H_{i-1} \quad(i=2, \ldots, n-1) \\
H_{1}=a B_{2} C_{1} B_{1} .
\end{array}\right.
$$

Then we define

$$
\begin{aligned}
k_{1} & =C_{1} B_{1}, \\
k_{2} & =C_{1} A_{1} B_{1}, \ldots, \\
k_{n-1} & =C_{1} A_{1}^{n-2} B_{1} .
\end{aligned}
$$

Obviously, $k_{i} \in \mathbb{R}^{1 \times 1}(i=1,2, \ldots,(n-1))$. Thus,

$$
M=\left(\begin{array}{cccc}
B_{1} & A_{1} B_{1} & A_{1}^{2} B_{1} & \ldots, \\
O & a k_{1} B_{2} & a\left(k_{2} B_{2}+k_{1} A_{2} B_{2}\right) & \ldots
\end{array}\right.
$$

$$
\left.\begin{array}{c}
A_{1}^{n-1} B_{1} \\
a\left(k_{n-1} B_{2}+k_{n-2} A_{2} B_{2}+\ldots+k_{1} A_{2}^{n-2} B_{2}\right)
\end{array}\right)
$$


Define function $f$ as

$$
\begin{aligned}
f(x ; i)= & k_{0} x^{i-1}+k_{1} x^{i-2}+k_{2} x^{i-3}+k_{3} x^{i-4}+\ldots \\
& +k_{i-3} x^{2}+k_{i-2} x+k_{i-1} x^{0}
\end{aligned}
$$

and note that $k_{0}=0$. Then

$$
\begin{gathered}
M=\left(\begin{array}{ccc}
B_{1} & A_{1} B_{1} & A_{1}^{2} B_{1} \\
a f\left(A_{2} ; 1\right) B_{2} & \text { af }\left(A_{2} ; 2\right) B_{2} & \text { af }\left(A_{2} ; 3\right) B_{2} \\
\ldots & A_{1}^{n-1} B_{1} \\
\ldots & \text { af }\left(A_{2} ; n\right) B_{2}
\end{array}\right) .
\end{gathered}
$$

Remark 2. It is shown that the constant $k_{i}$ is determined by matrices $A_{1}, B_{1}$, and $C_{1}$. Consequently, $f\left(A_{2} ; i\right)$ is determined not only by $A_{2}$, but also by $A_{1}, B_{1}$, and $C_{1}$. Then the controllability matrix $M$ is related to $A_{i} B_{i}(i=$ $1,2)$ and $C_{1}$ but is independent of $C_{2}$, which is the output matrix of the output linear subsystem $L_{2}$.

3.2. Observability matrix. Similarly, the observability matrix of the system can be defined by:

$$
\begin{gathered}
N=\left(\begin{array}{c}
C \\
C A \\
C A^{2} \\
\vdots \\
C A^{n-1}
\end{array}\right)=\left(\begin{array}{cc}
O & C_{2} \\
G_{1} & C_{2} A_{2} \\
G_{2} & C_{2} A_{2}^{2} \\
\vdots & \vdots \\
G_{n-1} & C_{2} A_{2}^{n-1}
\end{array}\right) \\
=\left(\begin{array}{cc}
O & C_{2} \\
a C_{2} B_{2} C_{1} & C_{2} A_{2} \\
G_{1} A_{1}+a C_{2} A_{2} B_{2} C_{1} & C_{2} A_{2}^{2} \\
\vdots & \vdots \\
G_{n-2} A_{1}+a C_{2} A_{2}^{n-2} B_{2} C_{1} & C_{2} A_{2}^{n-1}
\end{array}\right)
\end{gathered}
$$

where $G_{i}(i=1,2, \ldots, n-1)$ is the corresponding subblock of $N$. This implies

$$
\left\{\begin{array}{l}
G_{i}=G_{i-1} A_{1}+a C_{2} A_{2}^{n-2} B_{2} C_{1} \quad(i=2, \ldots, n-1), \\
G_{1}=a C_{2} B_{2} C_{1} .
\end{array}\right.
$$

Set $l_{i}=C_{2} A_{2}^{i-1} B_{2}(i=1,2, \ldots n-1)$ and $\left(l_{i} \in \mathbb{R}^{1 \times 1}\right)$. Then

$$
N=\left(\begin{array}{cc}
O & C_{2} \\
a l_{1} C_{1} & C_{2} A_{2} \\
a\left(l_{2} C_{1}+l_{1} C_{1} A_{1}\right) & C_{2} A_{2}^{2} \\
\vdots & \vdots \\
a\left(l_{n-1} C_{1} A_{1}^{0}+\ldots+l_{1} C_{1} A_{1}^{n-2}\right) & C_{2} A_{2}^{n-1}
\end{array}\right)
$$

$$
=\left(\begin{array}{cc}
O & C_{2} \\
a C_{1}\left(l_{1} E\right) & C_{2} A_{2} \\
a C_{1}\left(l_{2} E+l_{1} A_{1}\right) & C_{2} A_{2}^{2} \\
\vdots & \vdots \\
a C_{1}\left(l_{n-1} E+\cdots+l_{1} A_{1}^{n-2}\right) & C_{2} A_{2}^{n-1}
\end{array}\right) .
$$

Defining the function $\varphi$ as

$$
\begin{aligned}
\varphi(x ; i)= & l_{0} x^{i-1}+l_{1} x^{i-2}+l_{2} x^{i-3}+l_{3} x^{i-4}+\ldots \\
& +l_{i-3} x^{2}+l_{i-2} x+l_{i-1} x^{0}
\end{aligned}
$$

and noting $l_{0}=0$ yield

$$
N=\left(\begin{array}{cc}
a C_{1} \varphi\left(A_{1} ; 1\right) & C_{2} \\
a C_{1} \varphi\left(A_{1} ; 2\right) & C_{2} A_{2} \\
a C_{1} \varphi\left(A_{1} ; 3\right) & C_{2} A_{2}^{2} \\
\vdots & \vdots \\
a C_{1} \varphi\left(A_{1} ; n\right) & C_{2} A_{2}^{n-1}
\end{array}\right) .
$$

From the controllability (observability) theory for linear systems, we have the following theorem.

Theorem 1. In a small neighborhood of an equilibrium point, the sandwich system with backlash $\Sigma$ shown in Fig. 1, is controllable (resp. observable) if and only if its controllability (resp. observability) matrix $M$ (resp. $N)$ has full rank.

Remark 3. It can be seen that the observability matrix $N$ is related to $A_{i}, C_{i}$ is $(i=1,2)$ and $B_{2}$ but is independent of $B_{1}$, which is the input matrix of the linear subsystem $L_{1}$.

Remark 4. The duality between controllability and observability in all linear systems holds also in the linearized sandwich system with backlash. Note that the sandwich system with backlash is $\Sigma$ but its dual system is $\Sigma^{d}$. Therefore, it is not difficult to deduce that a realization of $\Sigma$ is observable (resp. controllable) if and only if the dual realization of $\Sigma^{d}$ is controllable (resp. observable).

\section{Observability and controllability analysis}

In this section, $M_{i}$ and $N_{i}(i=1,2)$ denote controllability and observability matrices, respectively. Furthermore, $\Sigma^{c}$ (resp. $\left.\Sigma^{o}\right)$ is defined as the controllable (observable) subspace of the sandwich system. Subsequently, $L_{i}^{c}$ and $L_{i}^{o}(i=1,2)$ are defined as the controllable and observable subspaces of the subsystems $L_{i}(i=1,2)$. Based on those premises, we have the following analysis. 
Case 1: $h^{*}$ is in memory zones.

In this case, $a=0$; then from (24) we obtain that

$$
M=\left(\begin{array}{ccccc}
B_{1} & A_{1} B_{1} & A_{1}^{2} B_{1} & \ldots & A_{1}^{n-1} B_{1} \\
O & O & O & \ldots & O
\end{array}\right) .
$$

Thus,

$$
\begin{aligned}
& \operatorname{rank} M=\operatorname{rank}\left(\begin{array}{ccccc}
B_{1} & A_{1} B_{1} & A_{1}^{2} B_{1} & \ldots & A_{1}^{n_{1}-1} B_{1}
\end{array}\right. \\
& \text {.. } \left.\quad A_{1}^{n-1} B_{1}\right) \text {. }
\end{aligned}
$$

Define the polynomial

$$
P(\lambda) \triangleq\left|\lambda E-A_{1}\right|=\lambda^{n_{1}}+a_{n_{1}-1} \lambda^{n_{1}-1}+\cdots+a_{0}
$$

as the characteristic polynomial of $A_{1}$. According to the Cayley-Hamilton theorem, we have

$$
A_{1}^{\mathrm{n}_{1}}+a_{n_{1}-1} A_{1}^{n_{1}-1}+\cdots+a_{0} E=0 .
$$

Accordingly,

$$
\begin{aligned}
A_{1}^{\mathrm{n}_{1}} B_{1}= & -a_{n_{1}-1} A_{1}^{n_{1}-1} B_{1}-a_{n_{1}-2} A_{1}^{\mathrm{n}_{1}-2} B_{1} \\
& -\cdots-a_{0} B_{1} .
\end{aligned}
$$

This states that vector $A_{1}^{\mathrm{n}_{1}} B_{1}$ can be linearly represented by column vectors $B_{1}, A_{1} B_{1}, \ldots, \quad A_{1}^{\mathrm{n}_{1}-1} B_{1}$, Furthermore, column vectors $A_{1}^{\mathrm{n}_{1}} B_{1}, A_{1}^{\mathrm{n}_{1}+1} B_{1}$, ..., $A_{1}^{n-1} B_{1}$ can be linearly represented by column vectors $B_{1}, A_{1} B_{1}, \ldots, A_{1}^{\mathrm{n}_{1}-1} B_{1}$.

In consequence, we have

$$
\begin{aligned}
\operatorname{rank}(M) & =\operatorname{rank}\left(\begin{array}{cccc}
B_{1} & A_{1} B_{1} & \ldots & A_{1}^{n_{1}-1} B_{1}
\end{array}\right) \\
& =\operatorname{rank}\left(M_{1}\right) .
\end{aligned}
$$

The expression (34) implies that in the case of $h^{*} \in$ $\left(-D_{2}, D_{1}\right)$ the dimension of the controllable subspace of the system $\Sigma$ is the same as for the input controllable subsystem $L_{1}$ in a small neighborhood of $h^{*}(k)$. Equivalently, we can say that $\operatorname{dim}\left(\Sigma^{c}\right)=\operatorname{dim}\left(L_{1}^{c}\right)$.

In the same way, from (29) we obtain that

$$
\operatorname{rank}(N)=\left(\begin{array}{cc}
O & C_{2} \\
O & C_{2} A_{2} \\
O & C_{2} A_{2}^{2} \\
\vdots & \vdots \\
O & C_{2} A_{2}^{n-1}
\end{array}\right)=\operatorname{rank}\left(\begin{array}{c}
C_{2} \\
C_{2} A_{2} \\
C_{2} A_{2}^{2} \\
\vdots \\
C_{2} A_{2}^{n-1}
\end{array}\right) .
$$

Note the characteristic polynomial of $A_{2}$ is

$$
\left|\lambda E-A_{2}\right|=\lambda^{n_{2}}+b_{n_{2}-1} \lambda^{n_{2}-1}+\cdots+b_{0} .
$$

By using the Cayley-Hamilton theorem, it can be proved that column vectors $C_{2} A_{2}^{n_{2}}, C_{2} A_{2}^{n_{2}+1}, \ldots, C_{2} A_{2}^{n-1}$ can be linearly represented by column vectors $C_{2}, C_{2} A_{2}, \ldots$, $C_{2} A_{2}^{n_{2}-1}$.
Thus, we derive

$$
\operatorname{rank}(N)=\operatorname{rank}\left(\begin{array}{c}
C_{2} \\
C_{2} A_{2} \\
C_{2} A_{2}^{2} \\
\vdots \\
C_{2} A_{2}^{n_{2}-1} \\
\vdots \\
C_{2} A_{2}^{n-1}
\end{array}\right)
$$

$$
=\operatorname{rank}\left(\begin{array}{c}
C_{2} \\
C_{2} A_{2} \\
C_{2} A_{2}^{2} \\
\vdots \\
C_{2} A_{2}^{n_{2}-1}
\end{array}\right)=\operatorname{rank}\left(N_{2}\right)
$$

The expression (37) indicates that in the case when $h^{*}$ is in memory zones the dimension of the observable subspace of the system $\Sigma$ is the same as the output observable subsystem $L_{2}$ in a small neighborhood of $h^{*}(k)$. That is, $\operatorname{dim}\left(\Sigma^{o}\right)=\operatorname{dim}\left(L_{2}^{o}\right)$.

\section{Case 2: $h^{*}$ is in linear zones.}

If $h^{*}$ is in increase zones, (14) implies that $a \in \partial v\left(h^{*}\right)=\left\{m_{1}\right\}$ or $\left\{0, m_{1}\right\} ; \quad$ then, letting $a=m_{1} \neq 0$, from (14),

$$
\begin{aligned}
& \operatorname{rank}(M) \\
& =\operatorname{rank}\left(\begin{array}{cc}
B_{1} & A_{1} B_{1} \\
m_{1} f\left(A_{2} ; 1\right) B_{2} & m_{1} f\left(A_{2} ; 2\right) B_{2}
\end{array}\right. \\
& \left.\begin{array}{ccc}
A_{1}^{2} B_{1} & \ldots & A_{1}^{n-1} B_{1} \\
m_{1} f\left(A_{2} ; 3\right) B_{2} & \ldots & m_{1} f\left(A_{2} ; n\right) B_{2}
\end{array}\right) \\
& =\operatorname{rank}\left(\begin{array}{cc}
B_{1} & A_{1} B_{1} \\
f\left(A_{2} ; 1\right) B_{2} & f\left(A_{2} ; 2\right) B_{2}
\end{array}\right. \\
& \left.\begin{array}{ll}
\ldots & A_{1}^{n-1} B_{1} \\
\ldots & A_{1}^{n-1} B_{1}
\end{array}\right) \\
& \geq \operatorname{rank}\left(\begin{array}{lllll}
B_{1} & A_{1} B_{1} & A_{1}^{2} B_{1} & \ldots & A_{1}^{n_{1}-1} B_{1}
\end{array}\right) \\
& =\operatorname{rank}\left(M_{1}\right)
\end{aligned}
$$

that is, $\operatorname{dim}\left(\Sigma^{c}\right) \geq \operatorname{dim}\left(L_{1}^{c}\right)$.

If $h^{*}$ is in decrease zones, (14) implies that $a \in \partial v\left(h^{*}\right)=\left\{m_{2}\right\}$ or $\left\{0, m_{2}\right\}$. Then, letting $a=m_{2} \neq 0$, similarly, we can obtain $\operatorname{rank}(M) \geq \operatorname{rank}\left(M_{1}\right)$. Therefore, when $h^{*}$ is in 


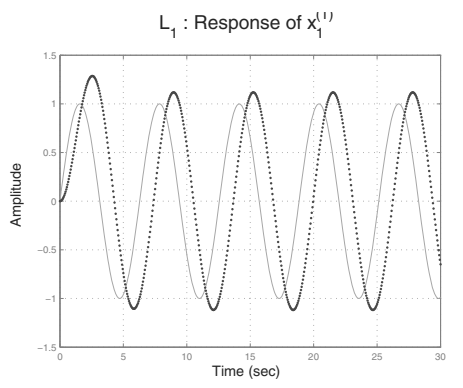

(a)

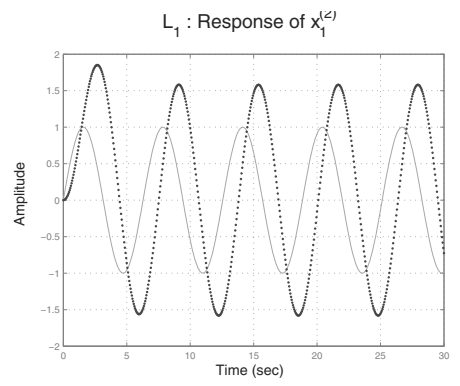

(b)

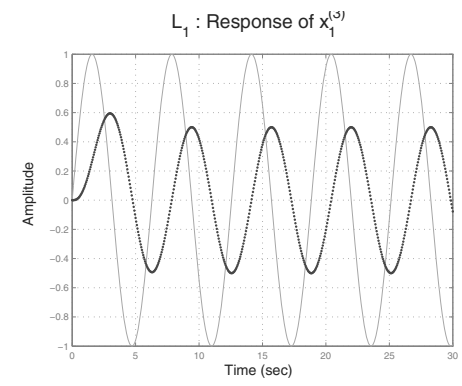

(c)

Fig. 2. State response of subsystem $L_{1}$.

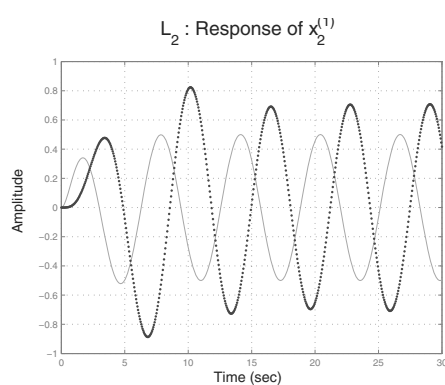

(a)

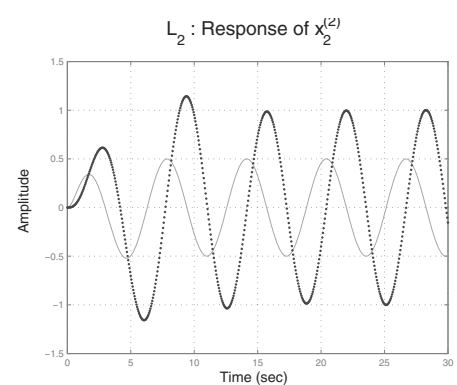

(b)

Fig. 3. State response of subsystem $L_{2}$ without the affection of backlash.

decrease zones, $\operatorname{dim}\left(\Sigma^{c}\right) \geq \operatorname{dim}\left(L_{1}^{c}\right)$ holds. Similarly, $\operatorname{dim}\left(\Sigma^{o}\right) \geq \operatorname{dim}\left(L_{2}^{o}\right)$.

Based on the above analysis, from (17)-(19), we have the following lemma.

Lemma 1. As for the sandwich system with backlash $\Sigma$ shown in Fig. 1, the following inequalities are satisfied:

$$
\operatorname{dim}\left(\Sigma^{c}\right) \geq \operatorname{dim}\left(L_{1}^{c}\right) \text { and } \operatorname{dim}\left(\Sigma^{o}\right) \geq \operatorname{dim}\left(L_{2}^{o}\right) .
$$

In particular, the equality holds if the equilibrium point is in memory zones.

Based on the lemma, we can prove the following theorem.

Theorem 2. For the sandwich system with backlash $\Sigma$ shown in Fig. 1,

(i) if the equilibrium point $h^{*}$ is in memory zones, $\Sigma$ is uncontrollable and unobservable;

(ii) if the equilibrium point $h^{*}$ is in linear zones, and if $\Sigma$ is controllable (observable), then its input and output linear subsystems $L_{i}(i=1,2)$ are all controllable (observable).

The proof can be found in Appendix.

Remark 5. In other cases the result is not true. In other words, even if $L_{i}(i=1,2)$ are all controllable (observable), we cannot guarantee that $L_{i}(i=1,2)$ are all controllable (observable).

\section{Numerical examples}

In this section, the proposed approach is used to analyze the observability and controllability of sandwich systems with backlash. Two numerical examples will be presented.

Example 1. In this example, $L_{1}$ and $L_{2}$, the linear dynamic subsystems, are respectively described by

$$
L_{1}:\left\{\begin{array}{l}
\dot{x}_{1}=\left(\begin{array}{ccc}
0 & 0 & -1 \\
1 & 0 & -3 \\
0 & 1 & -3
\end{array}\right) x_{1}+\left(\begin{array}{l}
1 \\
1 \\
0
\end{array}\right) u \\
h=\left(\begin{array}{ccc}
1 & 0 & -2
\end{array}\right) x_{1}
\end{array}\right.
$$

and

$$
L_{2}:\left\{\begin{array}{l}
\dot{x}_{2}=\left(\begin{array}{cc}
-1 & 1 \\
-1 & 0.5
\end{array}\right) x_{2}+\left(\begin{array}{l}
0 \\
1
\end{array}\right) v, \\
y=\left(\begin{array}{ll}
0 & 1
\end{array}\right) x_{2}
\end{array}\right.
$$

Suppose that the parameters of backlash in the system are $m_{1}=0.3, m_{2}=0.5, D_{1}=0.4, D_{2}=0.2$, Then, by (19), the state-space equations for the sandwich system with backlash by linearization are

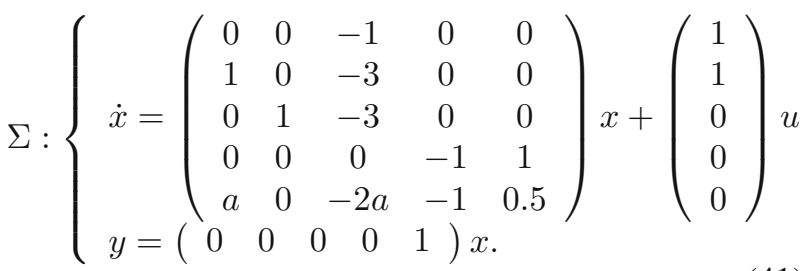


Based on (24) and (29),

$$
\begin{gathered}
M=\left(\begin{array}{ccccc}
1 & 0 & -1 & 2 & -3 \\
1 & 1 & -3 & 5 & -7 \\
0 & 1 & -2 & 3 & -4 \\
0 & 0 & 1 & -2.5 a & 3.75 a \\
0 & a & -1.5 a & 1.25 a & -0.875 a
\end{array}\right), \quad(42) \\
N=\left(\begin{array}{ccccc}
0 & 0 & 0 & 0 & 1 \\
a & 0 & -2 a & -1 & 0.5 \\
0.5 a & -2 a & 4 a & 0.5 & -0.75 \\
-2.75 a & 4 a & -5 a & 0.25 & 0.125 \\
4.125 a & -5 a & 5.5 a & -0.375 & 0.3125
\end{array}\right) .
\end{gathered}
$$

Obviously, the input linear subsystem $L_{1}$ is uncontrollable but observable, and the output linear subsystem $L_{2}$ is controllable and observable.

Suppose $u=\sin t$ and $x_{1}(0)=0$ if we take the output of subsystem $L_{1}$ as the input directly to subsystem $L_{2}$; the state responses of $L_{1}$ and $L_{2}$ are shown in Figs. 2 and 3, and the dotted line denotes the corresponding system response. Notice that $x_{i}^{(j)}$ means the $j$-th component of state vector $x_{i}$ for $L_{i}$. Moreover, from the state equations of $L_{2}$, we know that Fig. 3(b) represents the output of the whole system $\Sigma$. Thus, both Fig. 2 and Fig. 3 illustrate the system response without the effect of backlash.

We now analyze the observability and controllability of this sandwich system with backlash $\Sigma$.

In the following, two cases will be discussed.

(i) Equilibrium point $\boldsymbol{h}^{*}$ is in linear zones.

In this case, $a \neq 0, \operatorname{rank}(M)=4, \operatorname{rank}(N)=4$, and the system $\sum$ is uncontrollable and unobservable.

Remark 6. In fact, from Theorem $2, \Sigma$ is obviously uncontrollable, since $L_{1}$ is uncontrollable.

\section{(ii) Equilibrium point $\boldsymbol{h}^{*}$ is in memory zones.}

In this case, $a=0$, and $\operatorname{rank}(M)=2, \operatorname{rank}(N)=2$, so the system $\Sigma$ is uncontrollable and unobservable.

Remark 7. With the use of Theorem 2, since the equilibrium point $h^{*}$ is in memory zones of backlash, $\Sigma$ is inevitably uncontrollable (unobservable). Moreover, though subsystems $L_{i}(i=1,2)$ are all observable, $\Sigma$ is unobservable when $h^{*}$ is in increase or decrease zones, which shows that the observability of $\Sigma$ is affected by backlash.

In Fig. 4, both the input and output of backlash are illustrated. The corresponding state response of subsystem $L_{2}$ is given in Fig. 5.

Remark 8. As can be seen in Fig. 4, while equilibrium point is in memory zones, the peak output of $v$ is cut to flat. The state information of the input system $L_{1}$ was distorted by backlash.

Remark 9. By comparing Fig. 5 with Fig. 3, it can be seen that the response of each state has changed completely in the cases with and without the affection of backlash. As the role played by the backlash, in Fig. 5, the control input $u$ cannot completely affect the state vector $x_{2}$ of the output linear subsystem, which demonstrates non-smooth characteristic.

Example 2. Let us consider the following system:

$$
L_{1}:\left\{\begin{array}{l}
\dot{x}_{1}=\left(\begin{array}{ccc}
0 & 0 & -1 \\
1 & 0 & -3 \\
0 & 1 & -3
\end{array}\right) \\
h=\left(\begin{array}{lll}
0 & 1 & -2
\end{array}\right) x_{1}
\end{array} x_{1}+\left(\begin{array}{l}
1 \\
1 \\
0
\end{array}\right) u,\right.
$$

and

$$
L_{2}:\left\{\begin{array}{l}
\dot{x}_{2}=\left(\begin{array}{cc}
-4 & 5 \\
1 & 0
\end{array}\right) x_{2}+\left(\begin{array}{c}
-5 \\
1
\end{array}\right) v, \\
y=\left(\begin{array}{ll}
1 & -1
\end{array}\right) x_{2} .
\end{array}\right.
$$

Obviously, both $L_{1}$ and $L_{2}$ are uncontrollable and unobservable.

Suppose that the parameters of backlash in the system are $m_{1}=0.7, m_{2}=0.8, D_{1}=0.5, D_{2}=0.1$. Then, by (19), the state-space equations of the sandwich system with backlash by linearization are

$$
\Sigma:\left\{\begin{aligned}
\dot{x}= & \left(\begin{array}{ccccc}
0 & 0 & -1 & 0 & 0 \\
1 & 0 & -3 & 0 & 0 \\
0 & 1 & -3 & 0 & 0 \\
0 & -5 a & 10 a & -4 & 5 \\
0 & a & -2 a & 1 & 0
\end{array}\right) x \\
& +\left(\begin{array}{l}
1 \\
1 \\
0 \\
0 \\
0
\end{array}\right) u, \\
y= & \left(\begin{array}{llll}
0 & 0 & 0 & -1
\end{array}\right) x .
\end{aligned}\right.
$$

Suppose that $u=\sin t$ and $x_{1}(0)=0$ if we take the output of $L_{1}$ as the input directly to $L_{2}$; the corresponding response curve is shown in Figs. 6 and 7.

By (19), the state-space equations of the sandwich system with backlash can be derived. Then we know that the sandwich system $\Sigma$ is uncontrollable and unobservable. Figure 8 shows the input and output of backlash while Fig. 9 presents the corresponding response of $L_{2}$.

Remark 10. By comparing Fig. 7 with Fig. 9, it is known that, as for backlash, the control input $u$ cannot completely affect the state $x_{2}$. Then, the output of $L_{2}$ will not be able to receive the complete information sent from subsystem $L_{1}$. 


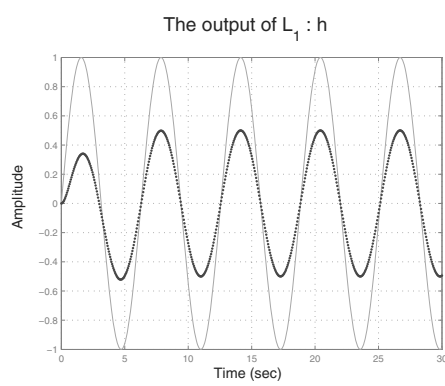

(a)

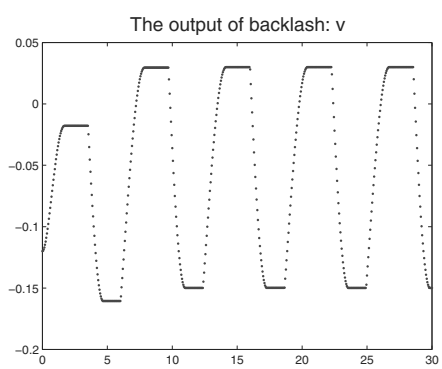

(b)

Fig. 4. Input and output of $L_{1}$ (a), output of backlash (b).

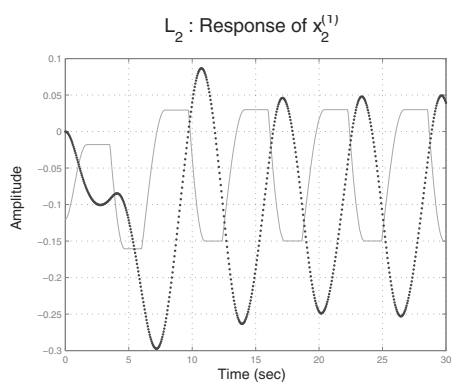

(a)

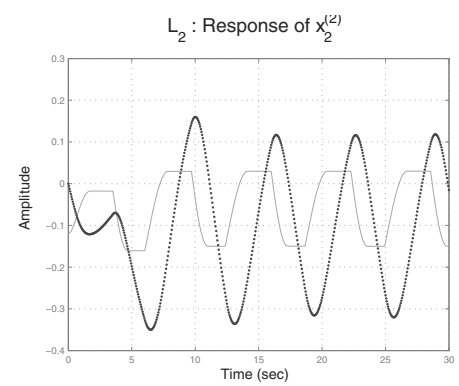

(b)

Fig. 5. State response of subsystem $L_{2}$ with the affection of backlash.

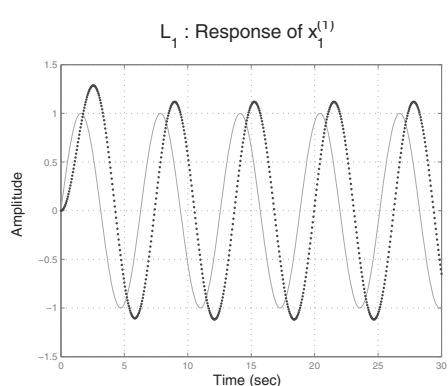

(a)

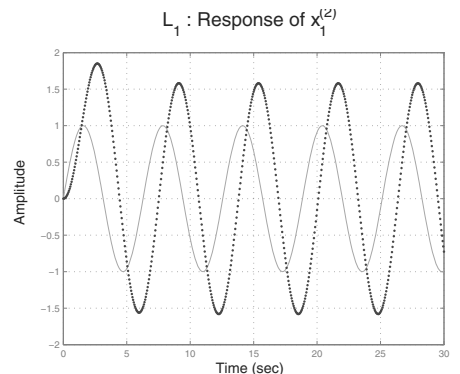

(b)

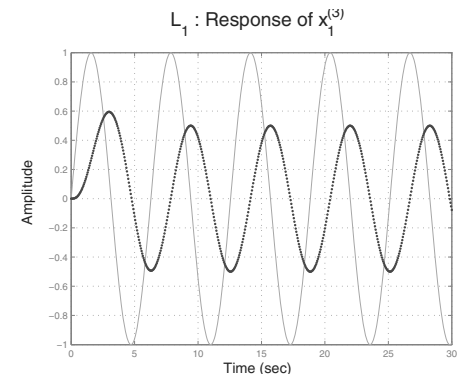

(c)

Fig. 6. State response of subsystem $L_{1}$.

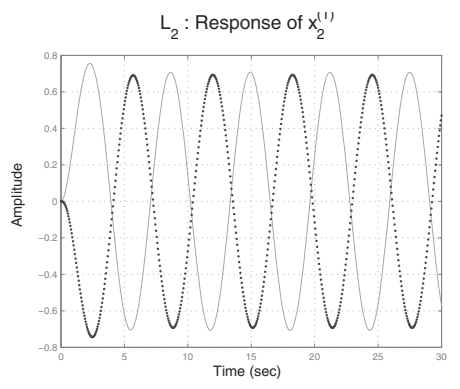

(a)

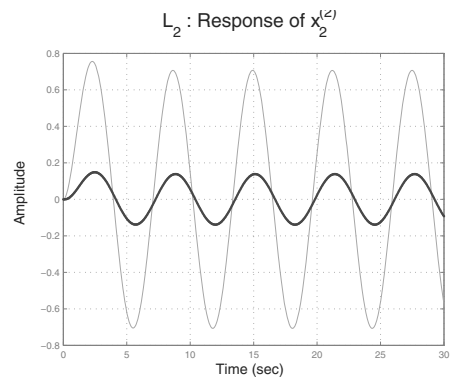

(b)

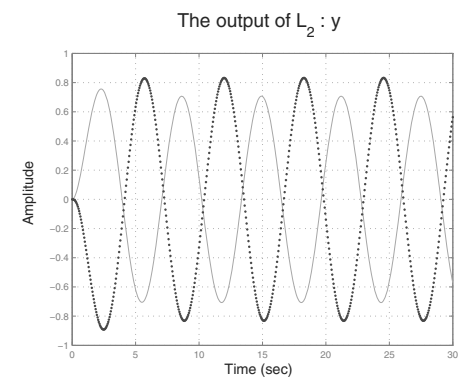

(c)

Fig. 7. State response of subsystem $L_{2}$ without the affection of backlash. 


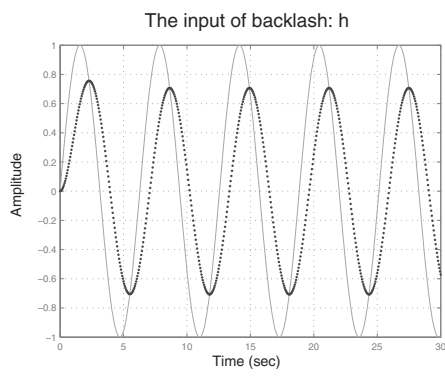

(a)

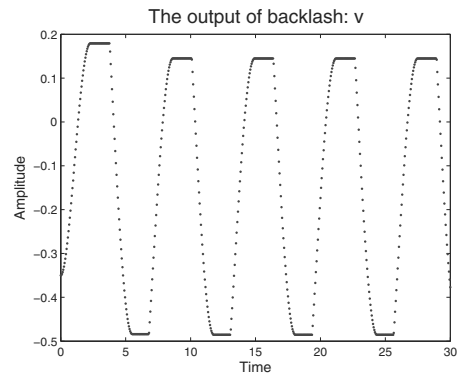

(b)

Fig. 8. Input and output of $L_{1}$ (a), output of backlash (b).

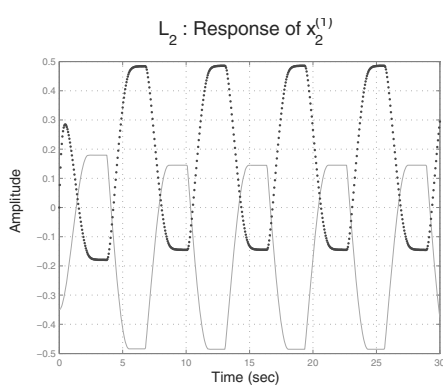

(a)

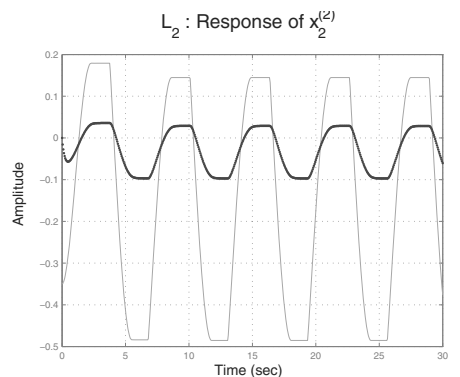

(b)

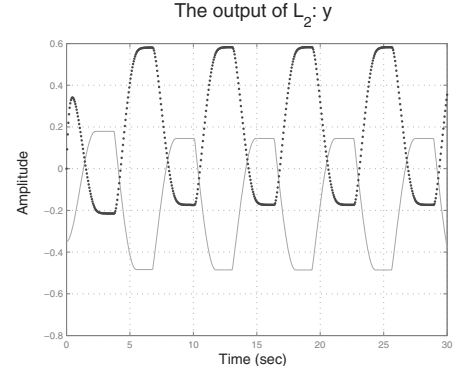

(c)

Fig. 9. State response of subsystem $L_{2}$ with the affection of backlash.

\section{Conclusion}

In this paper, a non-smooth state-space function was proposed for sandwich systems with backlash. In order to analyze the observability and controllability of sandwich systems with backlash, a linearization technique based on non-smooth optimization was employed to linearize non-smooth state-space function. This paper gives not only a sufficient condition for sandwich systems to be observable and controllable within a bounded region around the equilibrium point, but also a necessary condition. Moreover, this paper makes an analysis of relationships between the dimension of the observable and controllable spaces of sandwich systems and their subsystems. Finally, numerical examples were presented to intuitively analyze how observability and controllability of the sandwich systems are influenced by the existence of backlash.

The analysis of the controllability of sandwich systems with backlash in this paper only concerns the case when the control variable is unconstrained. However, in control engineering, the control variable is often constrained. In this case, the controllability problem becomes whether sandwich systems with backlash and input saturation are controllable. Obviously, it is an even more complex and interesting topic to be investigated and will be our research task in the future.

\section{Acknowledgment}

This research was partially supported by the National Science Foundation of China (NSFC Grants No. 61571302, 61371145, 61203108 and 61171088), the International Collaboration Project of Science and Technology Commission of Shanghai (Grants No. 14ZR1430300, 14140711200) and a Sino-Poland Cooperative Project (No. 35-23).

\section{References}

Balachandran, K. and Shanmugam, D. (2014). Controllability of nonlinear implicit fractional integrodifferential systems, International Journal of Applied Mathematics and Computer Science 24(4): 713-722, DOI: 10.2478/amcs-2014-0052.

Clarke, F.H. (1983). Optimization and Nonsmooth Analysis, John Wiley, New York, NY.

Dong, R., Tan, Y., Chen, H. and Xie, Y. (2012). Nonsmooth recursive identification of sandwich systems with backlash-like hysteresis, Journal of Applied Mathematics 2012: 1-16, ID 457601.

Herman, R. and Krener, A. (1977). Nonlinear controllability and observability, IEEE Transactions on Automatic Control 22(5): 728-740.

Isidori, A. (1989). Nonlinear Control Systems, Springer, London. 
Jank, G. (2002). Controllability, observability and optimal control of continuous-time 2-D systems, International Journal of Applied Mathematics and Computer Science 12(2): 181-195.

Kalman, R., Falb, P. and Arbib, M. (1969). Topics in Mathematical System Theory, Mc Graw-Hill Company, New York, NY.

Karthikeyan, S., Balachandran, K. and Murugesan, S. (2015). Controllability of nonlinear stochastic systems with multiple time-varying delays in control, International Journal of Applied Mathematics and Computer Science 25(2): 207-215, DOI: 10.1515/amcs-2015-0015.

Klamka, J. (1975). On the global controllability of perturbed nonlinear systems, IEEE Transactions on Automatic Control 20(1): 170-172.

Klamka, J. (1991). Controllability of Dynamical Systems, Kluwer Academic Publishers, Dordrecht.

Klamka, J. (2002). Controllability of nonlinear discrete systems, American Control Conference, Anchorage, AK, USA, pp. 4670-4671.

Klamka, J. (2013a). Constrained controllability of second order dynamical systems with delay, Control and Cybernetics 42(1): 111-121.

Klamka, J. (2013b). Controllability of dynamical systems: A survey, Bulletin of the Polish Academy of Sciences: Technical Sciences 61(2): 221-229.

Klamka, J., Czornik, A. and Niezabitowski, M. (2013). Stability and controllability of switched systems, Bulletin of the Polish Academy of Sciences: Technical Sciences 61(3): 547-554.

Koplon, R. and Sontag, E. (1993). Linear systems with sign observations, SIAM Journal Control and Optimization 31(12): 1245-1266.

Mincheko, L. and Sirotko, S. (2002). Controllability of non-smooth discrete systems with delay, Optimization 51(1): 161-174.

Murphey, T. and Burdick, J. (2002). Nonsmooth controllability theory and an example, 41st IEEE Conference on Decision and Control, Las Vegas, NV, USA, pp. 370-376.

Nordin, M. and Gutman, P.O. (2002). Controlling mechanical systems with backlash —a survey, Automatica 38(4): 1633-1649.

Qi, L. and Sun, J. (1993). A nonsmooth version of Newton's method, Mathematical Programming 58(3): 353-367.

Rockafellar, R.T. and Wets, R. J.B. (1998). Variational Analysis, Springer, Berlin.

Sontag, E. (1979). On the observability of polynomial systems, I: Finite-time problems, SIAM Journal of Control and Optimization 17(1): 139-151.

Sussmann, H. (1979). Single-input observability of continuous-time systems, Mathematical Systems Theory 12(3): 371-393.

van der Schaft, A.J. (1982). Observability and controllability for smooth nonlinear systems, SIAM Journal of Control and Optimization 20(3): 338-354.
Zhirabok, A. and Shumsky, A. (2012). An approach to the analysis of observability and controllability in nonlinear systems via linear methods, International Journal of Applied Mathematics and Computer Science 22(3): 507-522, DOI: 10.2478/v10006-012-0038-1.

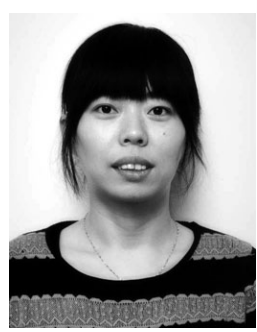

Na Luo obtained her Master's degree in mathematics from Shanghai Normal University in 2007. Presently, she is a Ph.D. candidate at the College of Mathematics and Science of the same university. She is also a lecturer at the School of General Education, Sanda University, Shanghai. Her research interests are in the analysis and control of non-linear dynamic systems.

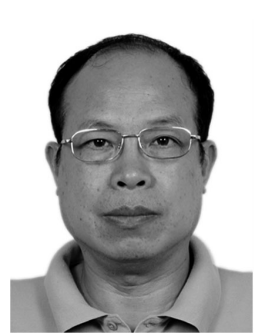

Yonghong Tan obtained the Ph.D. degree in electrical engineering from the University of Ghent, Belgium, in 1996. He has been a postdoctoral fellow with Simon Fraser University, Vancouver, BC, Canada, a visiting professor with Colorado State University, Fort Collins, USA, with Concordia University, Montreal, QC, Canada, with the Shibaura Institute of Technology, Japan, and with the University of Windsor, Canada. He has held professorships at the Guilin University of Electronic Technology, Guilin, China, and the University of Electronic Science and Technology of China, Chengdu. He is currently a professor at the College of Information, Mechanical and Electrical Engineering, Shanghai Normal University, China. He is the author and coauthor of more than 220 publications in peer-review journals, referenced conferences, and book chapters. He is also the holder of 11 patents. His research interests include modeling and control of nonlinear systems, mechatronics, intelligent control, and signal processing.

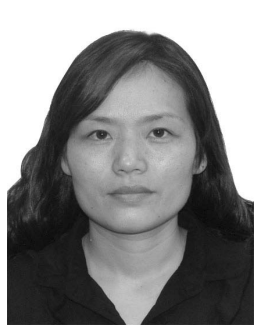

Ruili Dong received the Ph.D. degree from Shanghai Jiaotong University, China, in 2009. She was with Illinois University at Chicago, USA, from 2012 to 2013 . She is currently an associate professor at the College of Information, Mechanical and Electrical Engineering, Shanghai Normal University. She is also a visiting professor at the Dresden University of Technology, Germany. She has published more than $50 \mathrm{pa}-$ pers in peer-review journals and referenced conferences. She also holds five patents. Her research interests include identification and control of mechatronics and non-linear systems.

\section{Appendix}

\section{Observability and controllability analysis}

In this appendix, the proof of Theorem 2 is given.

Consider the two cases below.

I. In the case of memory zones, by virtue of Lemma 1, it holds that

$$
r(M)=r\left(M_{1}\right) \leq n_{1}<n_{1}+n_{2}=n .
$$

That is, $r(M)<n$, so $\Sigma$ is uncontrollable. 
Similarly,

$$
r(N)=r\left(N_{2}\right) \leq n_{2}<n_{1}+n_{2}=n,
$$

so $\Sigma$ is unobservable.

II. In the case of linear zones, if $\Sigma$ is controllable, by (22), we have

$\operatorname{rank}(M)$

$$
\begin{gathered}
=\operatorname{rank}\left(\begin{array}{ccc}
B_{1} & A_{1} B_{1} & A_{1}^{2} B_{1} \\
O & a k_{1} B_{2} & a\left(k_{2} B_{2}+k_{1} A_{2} B_{2}\right) \\
& \\
\ldots & a\left(k_{n-1} B_{2}+k_{n-2} A_{2} B_{2}+\ldots+k_{1} A_{2}^{n-2} B_{2}\right)
\end{array}\right) \\
\quad \ldots \quad
\end{gathered}
$$$$
=n \text {. }
$$

Then

$$
\operatorname{rank}\left(\begin{array}{ccccc}
B_{1} & A_{1} B_{1} & A_{1}^{2} B_{1} & \ldots & A_{1}^{n-1} B_{1}
\end{array}\right)=n_{1} .
$$

From (34), we then have

$$
\operatorname{rank}\left(M_{1}\right)=\operatorname{rank}(M)=n_{1}
$$

that is, the input linear subsystem $L_{1}$ is controllable.

On the other hand, we deduce that the output subsystem $L_{2}$ is also controllable: if $\Sigma$ is controllable, then

$$
\begin{aligned}
& \operatorname{rank}\left(\begin{array}{ccc}
0 & a k_{1} B_{2} & a\left(k_{2} B_{2}+k_{1} A_{2} B_{2}\right)
\end{array} \ldots\right. \\
& \left.a\left(k_{n-1} B_{2}+k_{n-2} A_{2} B_{2}+\ldots+k_{1} A_{2}^{n-2} B_{2}\right)\right) \\
& =n_{2} \text {. }
\end{aligned}
$$

Since the column vectors

$$
\begin{gathered}
0, \quad a k_{1} B_{2}, \quad a\left(k_{2} B_{2}+k_{1} A_{2} B_{2}\right), \quad \ldots \\
a\left(k_{n-1} B_{2}+k_{n-2} A_{2} B_{2}+\ldots+k_{1} A_{2}^{n-2} B_{2}\right)
\end{gathered}
$$

can be linearly represented by the column vectors

$$
B_{2}, \quad A_{2} B_{2}, \quad A_{2}^{2} B_{2}, \quad \ldots, \quad A_{2}^{n-2} B_{2},
$$

we derive

$$
\begin{aligned}
& n_{2} \leq \operatorname{rank}\left(B_{2} \quad A_{2} B_{2} \quad A_{2}^{2} B_{2} \quad \ldots \quad A_{2}^{n-2} B_{2}\right) \\
& =\operatorname{rank}\left(B_{2} \quad A_{2} B_{2} \quad \ldots \quad A_{2}^{\left(n_{2}-1\right)+\left(n_{1}-1\right)} B_{2}\right) .
\end{aligned}
$$

Define the polynomial

$$
P(\lambda) \triangleq\left|\lambda E-A_{2}\right|=\lambda^{\mathrm{n}_{2}}+d_{n_{2}-1} \lambda^{\mathrm{n}_{2}-1}+\ldots+d_{0}
$$

as the characteristic polynomial of $A_{2}$. By using the Cayley-Hamilton theorem, it can be proved that the column vectors $A_{2}^{\left(n_{2}-1\right)+1} B_{2}, A_{2}^{\left(n_{2}-1\right)+2} B_{2}, \ldots$, $A_{2}^{\left(n_{2}-1\right)+\left(n_{1}-1\right)} B_{2}$ can be linearly represented by the column vectors $B_{2}, A_{2} B_{2}, \ldots, A_{2}^{n_{2}-1} B_{2}$.

In consequence, we can deduce that

$$
\begin{aligned}
& n_{2} \leq \operatorname{rank}\left(B_{2} \quad A_{2} B_{2} \quad \ldots \quad A_{2}^{\left(n_{1}-1\right)+\left(n_{2}-1\right)} B_{2}\right) \\
& =\operatorname{rank}\left(B_{2} \quad A_{2} B_{2} \quad \ldots \quad A_{2}^{\left(n_{2}-1\right)} B_{2}\right) \\
& =\operatorname{rank}\left(M_{2}\right)
\end{aligned}
$$

because $\operatorname{rank}\left(M_{2}\right) \leq n_{2}$, which makes $\operatorname{rank}\left(M_{2}\right)=n_{2}$. That is, $L_{2}$ is controllable.

Thus, it can be concluded that $L_{i}(i=1,2)$ are controllable if $\Sigma$ is controllable. Similarly, it can be proved that $L_{i}(i=1,2)$ are observable if $\Sigma$ is observable.

Received: 24 July 2014 Revised: 10 April 2015 